\title{
Localized Thickening of a Compressed Elastic Band
}

\author{
B. BUFFONI ${ }^{1, \star}$ and S. REY ${ }^{2}$ \\ ${ }^{1}$ Ecole Polytechnique Fédérale de Lausanne (EPFL) Institute of Analysis and Scientific \\ Computing,CH-1015, Lausanne, Switzerland.E-mail:Boris.Buffoni@epfl.ch \\ ${ }^{2}$ rue du Simplon 15, CH-1006, Lausanne, Switzerland.E-mail: rey1707@yahoo.com
}

Received 14 December 2004; in revised form 01 August 2005

\begin{abstract}
An infinite elastic band is compressed along its unbounded direction, giving rise to a continuous family of homogeneous configurations that is parameterized by the compression rate $\beta<1$ ( $\beta=1$ when there is no compression). It is assumed that, for some critical value $\beta_{0}$, the compression force as a function of $\beta$ has a strict local extremum and that the linearized equation around the corresponding homogeneous configuration is strongly elliptic. Under these conditions, there are nearby localized deformations that are asymptotically homogeneous. When the compression force reaches a strict local maximum at $\beta_{0}$, they describe localized thickening and they occur for values of $\beta$ slightly smaller than $\beta_{0}$. Since the material is supposed to be hyperelastic, homogeneous and isotropic, the localized deformations are not due to localized imperfections. The method follows the one developed by A. Mielke for an elastic band under traction: interpretation of the nonlinear elliptic system as an infinite dimensional dynamical system in which the unbounded direction plays the role of time, its reduction to a center manifold and the existence of a homoclinic solution to the reduced finite dimensional problem in [A. Mielke, Hamiltonian and Lagrangian fiows on center manifolds, Lecture Notes in Mathematics 1489. Springer, Berlin Heidelberg New York, 1991]. The main difference lies in the fact that Agmon's condition does not hold anymore and therefore the linearized problem cannot be analyzed as in Mielke's work.
\end{abstract}

Mathematics Subject Classifications (2000): 76B20, 35J55, 37L10, 34C37.

Key words: Agmon's condition, center-manifold reduction, homoclinic solutions, localized structures, nonlinear elasticity, nonlinear elliptic systems, strong ellipticity, the complementing condition.

\section{Introduction}

We consider an elastic band that is isotropic and homogeneous, and that fills $\Omega=(0,1) \times \mathbb{R}$ when no force acts on it (natural configuration). Its deformations $\phi=\left(\phi_{1}, \phi_{2}\right): \Omega \rightarrow \mathbb{R}^{2}$ satisfy the equilibrium equation

$$
\operatorname{div}\left(\frac{\partial \bar{W}}{\partial F}(\nabla \phi)\right)=0 \text { on } \Omega
$$

$\star$ Corresponding author. 
where $\bar{W}$ is the density of elastic energy, which is a function of the deformation gradient $F=\nabla \phi($ a $(2 \times 2)$-matrix $)$. We assume that the boundary $\{0\} \times \mathbb{R}$ remains globally invariant under the considered deformations, there is no tangential force acting on $\{0\} \times \mathbb{R}$ and no force at all acting on the boundary $\{1\} \times \mathbb{R}$. This can be written

$$
\begin{cases}\phi_{1}(0, t)=0, & \frac{\partial \bar{W}}{\partial F_{21}}(\nabla \phi(0, t))=0, \\ \frac{\partial \bar{W}}{\partial F_{11}}(\nabla \phi(1, t))=0, & \frac{\partial \bar{W}}{\partial F_{21}}(\nabla \phi(1, t))=0,\end{cases}
$$

for all $t \in \mathbb{R}$. We shall use $x$ for the first variable and use $t$ for the second one. In this work, $t$ is a spatial variable; indeed we are only interested in elastostatic. However we use the notation $t$ because we shall consider equation (1) as a dynamical system with respect to $t$, in which $x$ is a 'mute' variable.

We are looking for solutions $\phi$ such that $\phi(\cdot, t) \in W^{2,2}(0,1)$ for all $t \in \mathbb{R}$ and the map $t \rightarrow \phi(\cdot, t)$ is $C^{2}$ for the $W^{2,2}(0,1)$ topology. In particular, $\phi \in C^{1}(\bar{\Omega})$ and we can therefore restrict ourselves to the case $\operatorname{det}(\nabla \phi)>0$ on $\bar{\Omega}$ and $\bar{W}$ smooth over a neighborhood of $\nabla \phi(\bar{\Omega})$. For every fixed $t \in \mathbb{R},(1)$ is understood to hold in $W^{2,2}(0,1)$.

As well known, the compression force

$$
P(\phi):=-\int_{0}^{1} \frac{\partial \bar{W}}{\partial F_{22}}(\nabla \phi) \mathrm{d} x
$$

is independent of $t$ for every solution $\phi$ to equations (1) and (2) that is not necessarily defined for all $t$, but at least on some rectangle $(0,1) \times\left(t_{1}, t_{2}\right)$ with $t_{1}$ $<t_{2}$. Indeed, integrate

$$
\partial_{x}\left\{\frac{\partial \bar{W}}{\partial F_{21}}(\nabla \phi)\right\}+\partial_{t}\left\{\frac{\partial \bar{W}}{\partial F_{22}}(\nabla \phi)\right\}=0
$$

over $(0,1) \times\left(s_{1}, s_{2}\right)$ with $t_{1}<s_{1}<s_{2}<t_{2}$. By $(2)$,

$$
\begin{aligned}
0 & =\int_{s_{1}}^{s_{2}} \int_{0}^{1} \partial_{t}\left\{\frac{\partial \bar{W}}{\partial F_{22}}(\nabla \phi(x, t))\right\} \mathrm{d} x \mathrm{~d} t \\
& =\int_{0}^{1} \frac{\partial \bar{W}}{\partial F_{22}}\left(\nabla \phi\left(x, s_{2}\right)\right) \mathrm{d} x-\int_{0}^{1} \frac{\partial \bar{W}}{\partial F_{22}}\left(\nabla \phi\left(x, s_{1}\right)\right) \mathrm{d} x .
\end{aligned}
$$

By isotropy, $\bar{W}(F)$ depends only on the singular values $\nu_{1}(F) \geq \nu_{2}(F)$ of $F$, that is, $\bar{W}(F)=W\left(\nu_{1}(F), \nu_{2}(F)\right)$ for some function

$$
W:\left\{\left(\mu_{1}, \mu_{2}\right) \in \mathbb{R}^{2}: \mu_{1} \geq \mu_{2}>0\right\} \rightarrow \mathbb{R},
$$


which we assume to be $C^{\infty}$ on $\left\{\left(\mu_{1}, \mu_{2}\right) \in \mathbb{R}^{2}: \mu_{1}>\mu_{2}>0\right\}$. We recall that the singular values of $F$ are the square roots of the eigenvalues of $F^{t} F$.

Instead of considering traction as A. Mielke in Chapter 10 in [14], we assume that (positive) compression along the $t$ axis gives rise to homogeneous deformations (that is, their derivatives are constant over $\Omega$ ). Like him, our aim is to study localized solutions, that is, solutions $\phi$ such that

$$
\lim _{|t| \rightarrow \infty}\left\|\nabla \phi(\cdot, t)-\nabla \phi_{\epsilon}(\cdot, t)\right\|_{W^{1,2}(0,1)}=0
$$

where $\phi_{\epsilon}=\left(\phi_{\epsilon, 1}, \phi_{\epsilon, 2}\right)$ is a homogeneous deformation near some particular homogeneous deformation $\phi_{0}$ around which the analysis is carried out. We follow the method in [14]: formulation as a dynamical system with respect to $t$, reduction to a center manifold, rescaling of the reduced equation and persistence of the homoclinic solution of the rescaled reduced equation.

The main new difficulties lie in the study of the linearized problem around $\phi_{0}$; indeed, Agmon's condition (see, e.g., [19]) fails, as it can be checked easily with the help of Mielke-Sprenger variational criterion (see the paragraph below just before the statement of Theorem 1). Agmon's condition is related to the coercivity of the quadratic part of the elastic energy near $\phi_{0}$ and regularity theory is then easier (see, e.g., [7]). Alternatively, regularity theory is available under the so-called complementing condition [1], and we devote an appendix to it. In Mielke's work, the elasticity tensor $C_{0}:=\partial^{2} \bar{W}\left(\nabla \phi_{0}\right) / \partial^{2} F$ is even non-negative, in the sense that $C_{0}[F, F] \geq 0$ for all matrices $F$ (equality occurs for some nonzero matrices). This property is very helpful to prove the absence of $t$-periodic eigenmodes. Indeed, integration by part leads to a first order differential equation that is much easier to deal with than the initial second order differential equation. In Mielke's setting, to check the complementing condition would probably be much easier too. In contrast, we are unable to avoid solving various second order ordinary differential equations.

Compression of finite rectangular elastic bands and three dimensional cylinders is considered in many papers (see, e.g., $[5,10,17,18]$ and their references). In [5], the rectangular elastic band is subjected to compression along an axis of symmetry. The surface compression at the ends has no tangential component, whereas the lateral surfaces (parallel to the axis) are stress-free. A solution is called of barreling type if it is axisymmetric, and of buckling type otherwise. Under appropriate conditions, to every compression rate $\lambda \in(0,1]$ corresponds of a homogeneous deformation $\mathbf{x}_{\lambda}$. For $\lambda$ near 1, $\mathbf{x}_{\lambda}$ is a weak local energy minimizer, but $\mathbf{x}_{\lambda}$ becomes unstable at some critical value $\lambda_{c} \in(0,1)$. In some cases, the associated instability corresponds to buckling and there is a critical value $\bar{\lambda} \in(0,1)$ such that all instabilities of buckling type occur above $\bar{\lambda}$ and all instabilities of barreling type occur below $\bar{\lambda}$. The complementing condition [1, 6, 19] fails at $\bar{\lambda}$ and Agmon's condition can fail if $\lambda<\bar{\lambda}$. 
Note that a solution of (1) and (2) can be extended to $(-1,1) \times \mathbb{R}$ by axial symmetry (that is, by anti-symmetry for $\phi_{1}$ and by symmetry for $\phi_{2}$ ), giving a deformation of barreling type on the (unbounded) strip $(-1,1) \times \mathbb{R}$. Hence a part of the analysis in [5] can be carried out in our present context. However, since the kind of instability we consider (that is, the pressure reaches a local extremum) is not explicitly mentioned in [5], we prefer to check directly the complementing condition for the linearized problem around $\phi_{0}$.

The boundary conditions (2) allow us to deal with a center manifold of dimension 3. Thanks to the existence of invariants, the final differential equation is two-dimensional, autonomous and totally integrable, like the one obtained by A. Mielke in [14]. Some of its integral curves are sketched in Figure 3 below. The closed curve starting at the origin corresponds to a localized deformation, whereas the other loops correspond to periodic deformations (their gradients are periodic in $t$ ). Extended by symmetry to $(-1,1) \times \mathbb{R}$, these periodic structures correspond to periodic solutions of barreling type and they are in spirit closer to the papers listed above on compression of finite rectangular bands. Non-integrable higher order ordinary differential equations describing localized patterns of elastic materials have attracted much attention in recent years (see, e.g., $[2,3,4,11,12])$. It would be nice to get examples in elasticity in which a center-manifold reduction leads to such non-integrable equations.

\section{Statement of the Problem}

We assume the existence of a homogeneous deformation of the form $\phi_{0}(x, t)=$ $\left(\alpha_{0} x, \beta_{0} t\right)$ with $0<\beta_{0}<\alpha_{0}$. We also suppose that the elasticity tensor $C_{0}:=$ $\partial^{2} \bar{W}\left(\nabla \phi_{0}\right) / \partial^{2} F$ is strongly elliptic:

$$
C_{0}\left[\xi_{1} \otimes \xi_{2}, \xi_{1} \otimes \xi_{2}\right]>0 \quad \text { for all } \quad \xi_{1}, \xi_{2} \in \mathbb{R}^{2} \backslash\{0\}
$$

Considering nearby homogeneous deformations of the form

$$
\nabla \phi=\left(\begin{array}{cc}
\alpha_{0}+a & b \\
c & \beta_{0}+d
\end{array}\right), \quad|a|,|b|,|c|,|d|<<1
$$

(where $a, b, c, d$ are constant), we get

$$
\begin{aligned}
& \left(\alpha_{0}+a\right)^{2}+b^{2}+c^{2}+\left(\beta_{0}+d\right)^{2} \\
& \quad=\left\{\nu_{1}(\nabla \phi)\right\}^{2}+\left\{\nu_{2}(\nabla \phi)\right\}^{2},\left(\alpha_{0}+a\right)\left(\beta_{0}+d\right)-b c=\nu_{1}(\nabla \phi) \nu_{2}(\nabla \phi),
\end{aligned}
$$




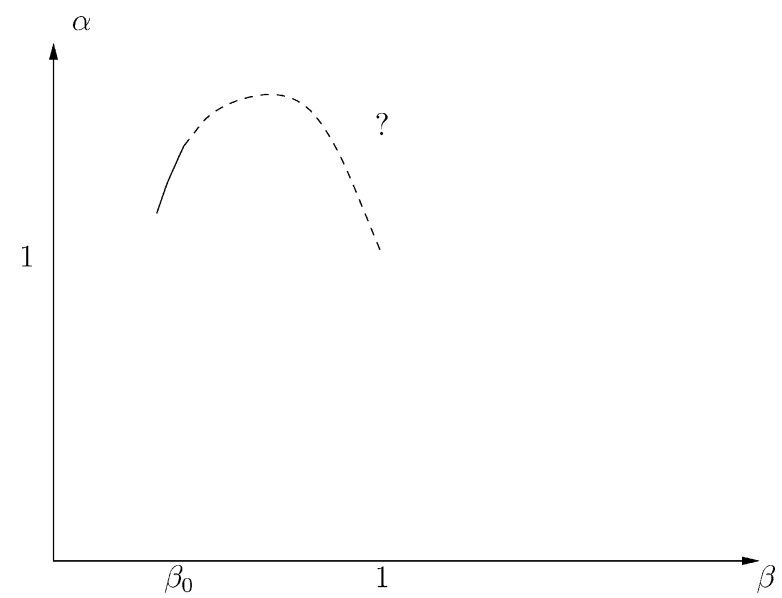

Figure 1. $\alpha=A(\beta)$.

and therefore

$$
\begin{aligned}
& \nu_{1}(\nabla \phi)=\alpha_{0}+a+O\left(a^{2}+b^{2}+c^{2}+d^{2}\right), \\
& \nu_{2}(\nabla \phi)=\beta_{0}+d+O\left(a^{2}+b^{2}+c^{2}+d^{2}\right) .
\end{aligned}
$$

This gives

$$
\begin{aligned}
W\left(\nu_{1}(\nabla \phi), \nu_{2}(\nabla \phi)\right)= & W\left(\alpha_{0}, \beta_{0}\right)+W_{1}\left(\alpha_{0}, \beta_{0}\right) a+W_{2}\left(\alpha_{0}, \beta_{0}\right) d \\
& +O\left(a^{2}+b^{2}+c^{2}+d^{2}\right) .
\end{aligned}
$$

Hence such a homogeneous configuration satisfies $W_{1}\left(\alpha_{0}, \beta_{0}\right)=0$ and the corresponding compression force on any cross-section is equal to $-W_{2}\left(\alpha_{0}, \beta_{0}\right)$, which is assumed to be positive.

Under the non-degeneracy hypothesis $W_{11}\left(\alpha_{0}, \beta_{0}\right) \neq 0$, there is locally near $\left(\alpha_{0}, \beta_{0}\right)$ a family of such configurations given by $\alpha=A(\beta)>\beta$ with $A\left(\beta_{0}\right)=\alpha_{0}$,

$$
W_{1}(A(\beta), \beta)=0 \quad \text { and } \quad A^{\prime}(\beta)=\frac{W_{12}(A(\beta), \beta)}{W_{11}(A(\beta), \beta)} \text { for all } \beta \text { near } \beta_{0},
$$

the compression force being

$$
\mathcal{P}(\beta):=-W_{2}(A(\beta), \beta)>0 .
$$

We shall see below that, as a consequence of strong ellipticity, $A^{\prime}\left(\beta_{0}\right)>0$ (see Figure 1).

We assume that there is an instability at $\left(\alpha_{0}, \beta_{0}\right)$ in the sense that

$$
0=\mathcal{P}^{\prime}\left(\beta_{0}\right)=-W_{21} A^{\prime}\left(\beta_{0}\right)-W_{22}=\frac{W_{12}^{2}}{W_{11}}-W_{22}
$$




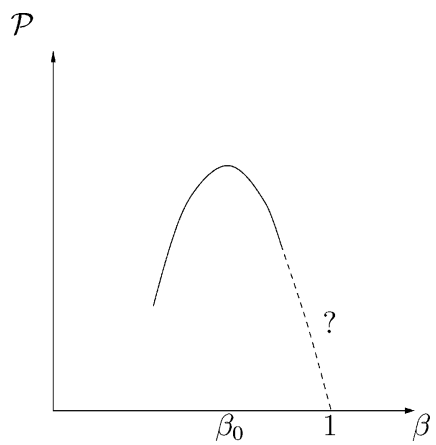

Figure 2. Here $\mathcal{P}^{\prime \prime}\left(\beta_{0}\right)<0$.

and

$$
\begin{aligned}
0 \neq \mathcal{P}^{\prime \prime}\left(\beta_{0}\right)= & \left(W_{12} / W_{11}\right)^{3} W_{111}-3\left(W_{12} / W_{11}\right)^{2} W_{112} \\
& +3\left(W_{12} / W_{11}\right) W_{122}-W_{222},
\end{aligned}
$$

where the derivatives of $W$ are taken at $\left(\alpha_{0}, \beta_{0}\right)$ (see Figure 2).

Since

$$
\nu_{1}(\nabla \phi)=\alpha_{0}+a+\frac{2 \beta_{0} b c+\alpha_{0}\left(b^{2}+c^{2}\right)}{2\left(\alpha_{0}^{2}-\beta_{0}^{2}\right)}+O\left(|a|^{3}+|b|^{3}+|c|^{3}+|d|^{3}\right)
$$

and

$$
\nu_{2}(\nabla \phi)=\beta_{0}+d-\frac{2 \alpha_{0} b c+\beta_{0}\left(b^{2}+c^{2}\right)}{2\left(\alpha_{0}^{2}-\beta_{0}^{2}\right)}+O\left(|a|^{3}+|b|^{3}+|c|^{3}+|d|^{3}\right),
$$

we get as in [14]

$$
\begin{aligned}
W\left(\nu_{1}(\nabla \phi), \nu_{2}(\nabla \phi)\right)= & W\left(\alpha_{0}, \beta_{0}\right)+W_{1}\left(\alpha_{0}, \beta_{0}\right) a+W_{2}\left(\alpha_{0}, \beta_{0}\right) d \\
& +\frac{1}{2}\left(r_{1} a^{2}+r_{2} b^{2}+r_{3} c^{2}+r_{4} d^{2}\right)+r_{5} b c \\
& +r_{6} a d+O\left(|a|^{3}+|b|^{3}+|c|^{3}+|d|^{3}\right)
\end{aligned}
$$

with $r_{1}=W_{11}\left(\alpha_{0}, \beta_{0}\right), r_{4}=W_{22}\left(\alpha_{0}, \beta_{0}\right), r_{6}=W_{12}\left(\alpha_{0}, \beta_{0}\right)$,

$$
r_{2}=r_{3}=\frac{\alpha_{0} W_{1}\left(\alpha_{0}, \beta_{0}\right)-\beta_{0} W_{2}\left(\alpha_{0}, \beta_{0}\right)}{\alpha_{0}^{2}-\beta_{0}^{2}}=\frac{-\beta_{0} W_{2}\left(\alpha_{0}, \beta_{0}\right)}{\alpha_{0}^{2}-\beta_{0}^{2}}
$$


and

$$
r_{5}=\frac{\beta_{0} W_{1}\left(\alpha_{0}, \beta_{0}\right)-\alpha_{0} W_{2}\left(\alpha_{0}, \beta_{0}\right)}{\alpha_{0}^{2}-\beta_{0}^{2}}=\frac{-\alpha_{0} W_{2}\left(\alpha_{0}, \beta_{0}\right)}{\alpha_{0}^{2}-\beta_{0}^{2}} .
$$

Strong ellipticity of the linearized problem at $\phi_{0}$ is equivalent to

$$
r_{1}, r_{2}, r_{3}, r_{4}>0 \text { and }\left|r_{5}+r_{6}\right|<\sqrt{r_{1} r_{4}}+\sqrt{r_{2} r_{3}}
$$

(see [13] or Section 10.2 in [14]), which is assumed. Thanks to (5), we have $W_{2}\left(\alpha_{0}, \beta_{0}\right)<0, r_{5}>r_{2}$ and, thanks to (6), $r_{6}^{2}=r_{1} r_{4}$. Therefore (9) is equivalent to

$$
r_{1}, r_{2}, r_{3}, r_{4}>0, r_{2}=r_{3}, r_{5}+2 r_{6}<r_{2}<r_{5} \text { and } r_{6}=-\sqrt{r_{1} r_{4}}<0 .
$$

As a consequence $A^{\prime}\left(\beta_{0}\right)>0$.

Still for the linearized problem at $\phi_{0}$, if equation (9) holds, then Agmon's condition for the Neumann boundary condition (that is, at the boundary $\{1\} \times \mathbb{R}$ ) is fulfilled exactly when

$$
r_{6}^{2} \sqrt{r_{3} r_{2}}+r_{5}^{2} \sqrt{r_{1} r_{4}} \leq \sqrt{r_{1} r_{4}} \sqrt{r_{3} r_{2}}\left(\sqrt{r_{1} r_{4}}+\sqrt{r_{3} r_{2}}\right)
$$

(see Theorem 5.1 in [16] and [8,9] for further developments). From $r_{6}^{2}=r_{1} r_{4}$ and $r_{5}^{2}>r_{3} r_{2}$, we deduce that Agmon's condition is not satisfied. In the traction case [14], all $r_{i}$ are positive, $r_{6}^{2}=r_{1} r_{4}$ and $r_{5}^{2}<r_{2} r_{3}$. Hence both strong ellipticity and Agmon's condition hold.

The following theorem is our main result. Its proof will be given in the remaining sections.

THEOREM 1. Fix $k \geq 2$. Under the above hypotheses, for all small $\epsilon>0$, there exist $\beta_{\epsilon}>0$ and a solution $\phi_{\epsilon}=\left(\phi_{\epsilon, 1}, \phi_{\epsilon, 2}\right)$ of (1) and (2), such that

1. $\phi_{\epsilon}(\cdot, t) \in W^{2,2}(0,1)$ for all $t \in \mathbb{R}$ and the map $t \rightarrow \phi_{\epsilon}(\cdot, t)$ is $C^{k}$ for the $W^{2,2}(0,1)$ topology,

2. for every fixed $t \in \mathbb{R}$, (1) holds in $W^{2,2}(0,1)$,

3. $K^{-1} \epsilon^{2}<\left|\beta_{\epsilon}-\beta_{0}\right|<K \epsilon^{2}$,

4. $\lim _{|t| \rightarrow \infty} \nabla \phi_{\epsilon}(\cdot, t)=\operatorname{diag}\left(A\left(\beta_{\epsilon}\right), \beta_{\epsilon}\right)$ in $W^{1,2}(0,1)$,

5. $K^{-1} \epsilon^{2}<\sup _{t \in \mathbb{R}}\left\|\nabla \phi_{\epsilon}(\cdot, t)-\operatorname{diag}\left(A\left(\beta_{\epsilon}\right), \beta_{\epsilon}\right)\right\|_{W^{1,2}(0,1)}<K \epsilon^{2}$,

6. $\lim _{|t| \rightarrow \infty} e^{\epsilon|t| / K}\left\|\nabla \phi_{\epsilon}(\cdot, t)-\operatorname{diag}\left(A\left(\beta_{\epsilon}\right), \beta_{\epsilon}\right)\right\|_{W^{1,2}(0,1)}=0$ (localized deformation),

7. $\phi_{\epsilon, 1}(x,-t) \equiv \phi_{\epsilon, 1}(x, t)$ and $\phi_{\epsilon, 2}(x,-t) \equiv-\phi_{\epsilon, 2}(x, t)$ (symmetry or 'reversibility'),

8. $\phi_{\epsilon, 1}(1,0)>A\left(\beta_{\epsilon}\right)$ exactly when $\mathcal{P}^{\prime \prime}\left(\beta_{0}\right)<0$ (localized thickening),

9. $\beta_{\varepsilon}<\beta_{0}$ exactly when $\mathcal{P}^{\prime \prime}\left(\beta_{0}\right)<0$,

where $K>0$ is independent of $\epsilon$. 
As an example, consider $W$ given by

$$
W(\alpha, \beta)=a(\alpha)+a(\beta)+b(\alpha \beta)
$$

with $a^{\prime} \geq 0$ and $a^{\prime \prime}, b^{\prime \prime}>0$. For fixed $0<\beta_{0}<\alpha_{0}$, we assume that $b^{\prime}\left(\alpha_{0} \beta_{0}\right)<0$ and $a^{\prime}\left(\alpha_{0}\right)+\beta_{0} b^{\prime}\left(\alpha_{0} \beta_{0}\right)=0$, so that

$$
W_{2}\left(\alpha_{0}, \beta_{0}\right)=a^{\prime}\left(\beta_{0}\right)+\alpha_{0} b^{\prime}\left(\alpha_{0} \beta_{0}\right)<a^{\prime}\left(\alpha_{0}\right)+\beta_{0} b^{\prime}\left(\alpha_{0} \beta_{0}\right)=W_{1}\left(\alpha_{0}, \beta_{0}\right)=0 .
$$

When $\alpha \rightarrow a(\alpha)$ is an affine function of $\alpha^{2}$ in a neighborhood of $\alpha=1, \bar{W}$ is smooth in a neighborhood of the identity. In such a case, the hypothesis that $\Omega$ corresponds to the natural configuration leads to

$$
W_{1}(1,1)=a^{\prime}(1)+b^{\prime}(1)=0 \text { and } W_{2}(1,1)=a^{\prime}(1)+b^{\prime}(1)=0 .
$$

Moreover

$$
\begin{aligned}
& r_{1}=a^{\prime \prime}\left(\alpha_{0}\right)+\beta_{0}^{2} b^{\prime \prime}\left(\alpha_{0}, \beta_{0}\right), r_{4}=a^{\prime \prime}\left(\beta_{0}\right)+\alpha_{0}^{2} b^{\prime \prime}\left(\alpha_{0}, \beta_{0}\right), \\
& r_{6}=b^{\prime}\left(\alpha_{0} \beta_{0}\right)+\alpha_{0} \beta_{0} b^{\prime \prime}\left(\alpha_{0}, \beta_{0}\right), r_{2}=r_{3}=\beta_{0}\left(\alpha_{0}^{2}-\beta_{0}^{2}\right)^{-1}\left|W_{2}\left(\alpha_{0}, \beta_{0}\right)\right|, \\
& r_{5}=\alpha_{0}\left(\alpha_{0}^{2}-\beta_{0}^{2}\right)^{-1}\left|W_{2}\left(\alpha_{0}, \beta_{0}\right)\right| .
\end{aligned}
$$

Let us recall some equations to be checked:

$$
\begin{aligned}
& a^{\prime} \geq 0, a^{\prime \prime}>0, b^{\prime \prime}>0,0<\beta_{0}<\alpha_{0}, b^{\prime}\left(\alpha_{0} \beta_{0}\right)<0 \\
& a^{\prime}\left(\alpha_{0}\right)+\beta_{0} b^{\prime}\left(\alpha_{0} \beta_{0}\right)=0, a^{\prime}(1)+b^{\prime}(1)=0, r_{5}+2 r_{6}<r_{2}, r_{6}=-\sqrt{r_{1} r_{4}}
\end{aligned}
$$

and equation (7), the other ones being obviously satisfied. The conditions $r_{5}+2 r_{6}$ $<r_{2}$ and $r_{6}=-\sqrt{r_{1} r_{4}}$ are fulfilled if $b^{\prime \prime}\left(\alpha_{0} \beta_{0}\right)$ and $a^{\prime}\left(\beta_{0}\right)$ are small enough with respect to $\left|b^{\prime}\left(\alpha_{0} \beta_{0}\right)\right|$, and if $a^{\prime \prime}\left(\alpha_{0}\right)$ and $a^{\prime \prime}\left(\beta_{0}\right)$ are chosen appropriately. All these conditions can be satisfied with $\alpha_{0}>1$ and $\alpha_{0} \beta_{0}<1$. Condition (7) can be fulfilled by chosen carefully $a\left(\alpha_{0}\right), a^{\prime \prime \prime}\left(\beta_{0}\right)$ and $b^{\prime \prime \prime}\left(\alpha_{0} \beta_{0}\right)$.

\section{Formulation Around $\phi_{0}$ as a Dynamical System}

Let

$$
\phi(x, t)=\left(\begin{array}{c}
\alpha_{0} x+u_{1}(x, t) \\
\beta_{0} t+\gamma(t)+u_{2}(x, t)
\end{array}\right),
$$

where $\left|u_{1}\right|, u_{2} \mid<<1$ and $\left\langle u_{2}\right\rangle:=\int_{0}^{1} u_{2}(x, t) \mathrm{d} x=0$. Writing ${ }^{\prime}=\frac{\mathrm{d}}{\mathrm{d} x}$ and $\cdot=\frac{\mathrm{d}}{\mathrm{d} t}$, we have

$$
\nabla \phi(x, t)=\left(\begin{array}{lr}
\alpha_{0}+u_{1}^{\prime}(x, t) & v_{1}(x, t) \\
u_{2}^{\prime}(x, t) & \beta_{0}+v_{2}(x, t)
\end{array}\right),
$$

with $\dot{u}_{1}=v_{1}, \dot{\gamma}=\left\langle v_{2}\right\rangle$ and $\dot{u}_{2}=v_{2}-\left\langle v_{2}\right\rangle$. 
Substituting (10) and (11) into (1) and (2), and using (8), the equilibrium equations become

$$
\left\{\begin{array}{l}
\dot{u}_{1}=v_{1} \\
\dot{u}_{2}=v_{2}-\left\langle v_{2}\right\rangle \\
\dot{v}_{1}=-\frac{r_{1}}{r_{2}} u_{1}^{\prime \prime}-\frac{r_{5}+r_{6}}{r_{2}} v_{2}^{\prime}+g_{1} \\
\dot{v}_{2}=-\frac{r_{2}}{r_{4}} u_{2}^{\prime \prime}-\frac{r_{5}+r_{6}}{r_{4}} v_{1}^{\prime}+g_{2}
\end{array}\right\} \text { on }(0,1) \times \mathbb{R},
$$

where $g_{1}, g_{2}=O\left(\left|u_{1}^{\prime \prime}\right|+\left|u_{2}^{\prime \prime}\right|+\left|v_{1}^{\prime}\right|+\left|v_{2}^{\prime}\right|\right) O\left(\left|u_{1}^{\prime}\right|+\left|u_{2}^{\prime}\right|+\left|v_{1}\right|+\left|v_{2}\right|\right), g_{1}$, $g_{2}$ are linear with respect to $\left(u_{1}^{\prime \prime}, u_{2}^{\prime \prime}, v_{1}^{\prime}, v_{2}^{\prime}\right)$ (for any "frozen" $\left(u_{1}^{\prime}, u_{2}^{\prime}, v_{1}, v_{2}\right)$ ), and $g_{3}, g_{4}=O\left(\left|u_{1}^{\prime}\right|^{2}+\left|u_{2}^{\prime}\right|^{2}+\left|v_{1}\right|^{2}+\left|v_{2}\right|^{2}\right)$. Note that $\gamma$ does not appear explicitly in (12).

The symmetry of (1) and (2) with respect to the $0 x$ axis leads to the reversibility of (12) with respect to $\left(u_{1}, u_{2}, v_{1}, v_{2}\right) \rightarrow\left(u_{1},-u_{2},-v_{1}, v_{2}\right)$. In particular, $g_{1}$ and $g_{4}$ are invariant under this symmetry, whereas the signs of $g_{2}$ and $g_{3}$ are changed.

To simplify the boundary conditions, we set

$$
p_{1}=r_{5} u_{2}^{\prime}+r_{2} v_{1}+\frac{r_{2}}{r_{5}} g_{3}, \quad p_{2}=r_{6} u_{1}^{\prime}+r_{4} v_{2}+\frac{r_{4}}{r_{6}} g_{4},
$$

so that equation (12) becomes

$$
\left\{\begin{array}{l}
\dot{u}_{1}=\frac{1}{r_{2}} p_{1}-\frac{r_{5}}{r_{2}} u_{2}^{\prime}+f_{1} \\
\dot{u}_{2}=\frac{1}{r_{4}} p_{2}-\frac{1}{r_{4}}\left\langle p_{2}\right\rangle-\frac{r_{6}}{r_{4}} u_{1}^{\prime}+\frac{r_{6}}{r_{4}} u_{1}(1)+f_{2} \\
\dot{p}_{1}=-\frac{r_{6}}{r_{4}} p_{2}^{\prime}+f_{3} \\
\dot{p}_{2}=-\frac{r_{5}}{r_{2}} p_{1}^{\prime}-\frac{r_{5}^{2}-r_{2}^{2}}{r_{2}} u_{2}^{\prime \prime}+f_{4} \\
u_{1}=0 \text { and }\left(r_{2}^{2}-r_{5}^{2}\right) u_{2}^{\prime}+r_{5} p_{1}=0 \text { on }\{0\} \times \mathbb{R}, \\
p_{2}=0 \text { and }\left(r_{2}^{2}-r_{5}^{2}\right) u_{2}^{\prime}+r_{5} p_{1}=0 \text { on }\{0\} \times \mathbb{R}, \\
\left\langle u_{2}\right\rangle=0
\end{array}\right.
$$

where $f_{1}, f_{2}=O\left(\left|u_{1}^{\prime}\right|^{2}+\left|u_{2}^{\prime}\right|^{2}+\left|p_{1}\right|^{2}+\left|p_{2}\right|^{2}\right)$,

$$
f_{3}, f_{4}=O\left(\left|u_{1}^{\prime \prime}\right|+\left|u_{2}^{\prime \prime}\right|+\left|p_{1}^{\prime}\right|+\left|p_{2}^{\prime}\right|\right) O\left(\left|u_{1}^{\prime}\right|+\left|u_{2}^{\prime}\right|+\left|p_{1}\right|+\left|p_{2}\right|\right)
$$

and $f_{3}, f_{3}$ are linear with respect to $\left(u_{1}^{\prime \prime}, u_{2}^{\prime \prime}, p_{1}^{\prime}, p_{2}^{\prime}\right)$ (for any "frozen" $\left(u_{1}^{\prime}, u_{2}^{\prime}\right.$, $\left.\left.p_{1}, p_{2}\right)\right)$.

Now equation (13) is reversible with respect to

$$
\left(u_{1}, u_{2}, p_{1}, p_{2}\right) \rightarrow\left(u_{1},-u_{2},-p_{1}, p_{2}\right):=\mathcal{R}\left(u_{1}, u_{2}, p_{1}, p_{2}\right) .
$$


Let $Z$ be the real Hilbert space

$$
\begin{aligned}
Z= & \left\{z=\left(u_{1}, u_{2}, p_{1}, p_{2}\right): u_{1}, u_{2} \in W^{1,2}(0,1), p_{1}, p_{2} \in L^{2}(0,1),\right. \\
& \left.u_{1}(0)=0,\left\langle u_{2}\right\rangle=0\right\}
\end{aligned}
$$

We define in $Z$ the operator $\mathcal{L}$ by

$$
\mathcal{L} z=\left(\begin{array}{l}
\frac{1}{r_{2}} p_{1}-\frac{r_{5}}{r_{2}} u_{2}^{\prime} \\
\frac{1}{r_{4}}\left(p_{2}-\left\langle p_{2}\right\rangle\right)-\frac{r_{6}}{r_{4}}\left(u_{1}^{\prime}-u_{1}(1)\right) \\
-\frac{r_{6}}{r_{4}} p_{2}^{\prime} \\
-\frac{r_{5}}{r_{2}} p_{1}^{\prime}+\frac{r_{5}^{2}-r_{2}^{2}}{r_{2}} u_{2}^{\prime \prime}
\end{array}\right),
$$

the domain of which being

$$
\begin{aligned}
D(\mathcal{L}) & =\left\{z=\left(u_{1}, u_{2}, p_{1}, p_{2}\right): u_{1}, u_{2} \in W^{2,2}(0,1), p_{1}, p_{2} \in W^{1,2}(0,1),\right. \\
\left\langle u_{2}\right\rangle & =0, u_{1}=0 \text { and } r_{5} p_{1}+\left(r_{2}^{2}-r_{5}^{2}\right) u_{2}^{\prime}=0 \text { at } x=0, \\
p_{2} & \left.=0 \text { and } r_{5} p_{1}+\left(r_{2}^{2}-r_{5}^{2}\right) u_{2}^{\prime}=0 \text { at } x=1\right\} .
\end{aligned}
$$

We endow $Z$ and $D(\mathcal{L})$ with the norms of the Sobolev spaces that appear in their definitions (the norm $\|z\|_{D(\mathcal{L})}$ so defined is equivalent to the norm $\left.\|z\|_{Z}+\|\mathcal{L} z\|_{Z}\right)$. The problem is to solve

$$
\dot{z}=\mathcal{L} z+f(z),
$$

where $f=\left(f_{1}, f_{2}, f_{3}, f_{4}\right)$ is well-defined and smooth as a map from some neighborhood of the origin in $D(\mathcal{L})$ to $Z$.

The following lemma is crucial and is proved in Appendices A and B. Here $Z$ is regarded as a complex Hilbert space.

LEMMA 2. The operator $\mathcal{L}$ satisfies the following properties:

1. The only eigenvalue on the imaginary axis is 0 .

2. The resolvant $(\mathcal{L}-i s)^{-1}: Z \rightarrow D(\mathcal{L})$ exists for all real numbers s such that $|s|$ is large enough, and it satisfies the estimate

$$
\left\|(\mathcal{L}-i s)^{-1}\right\|_{Z \rightarrow Z}=O(1 /|s|) \quad \text { as } \quad|s| \rightarrow \infty .
$$


COROLLARY 3. The spectrum of $\mathcal{L}$ is discrete (that is, consists of isolated eigenvalues of finite multiplicities).

Proof. Let $s \in \mathbb{R}$ be such that $|s|$ is large enough. Since the resolvant $(\mathcal{L}-i s)^{-1}: Z \rightarrow Z$ is bounded, it is also bounded as an operator from $Z$ to $D(\mathcal{L})$. Since the inclusion $D(\mathcal{L}) \subset Z$ is compact, the resolvant $(\mathcal{L}-i s)^{-1}: Z \rightarrow Z$ is compact. Hence the spectrum of $(\mathcal{L}-i s)^{-1}$ is discrete with the exception of the value 0 , and thus the spectra of $\mathcal{L}-i s$ and $\mathcal{L}$ are discrete.

The generalized kernel of $\mathcal{L}$ is finite dimensional. It can be computed explicitly and it is found to be equal to $\operatorname{span}\left(\psi_{1}, \psi_{2}, \psi_{3}\right)$ with

$$
\begin{aligned}
& \psi_{1}=\left(\begin{array}{l}
x \\
0 \\
0 \\
0
\end{array}\right), \psi_{2}=\frac{1}{6 r_{2}}\left(\begin{array}{c}
0 \\
-r_{5}\left(3 x^{2}-1\right) \\
6\left(r_{2}^{2}-r_{5}^{2}\right) x \\
0
\end{array}\right), \\
& \psi_{3}=\frac{1}{6 r_{1} r_{2}}\left(\begin{array}{c}
\left(r_{2}^{2}-r_{2}^{2}+r_{5} r_{6}\right) x^{3} \\
0 \\
0 \\
-3 r_{6}\left(r_{2}^{2}-r_{5}^{2}\right)\left(x^{2}-1\right)
\end{array}\right),
\end{aligned}
$$

with $\mathcal{L} \psi_{1}=0, \mathcal{L} \psi_{2}=\psi_{1}, \mathcal{L} \psi_{3}=\psi_{2}$. The projection $\Pi: Z \rightarrow \operatorname{span}\left(\psi_{1}, \psi_{2}, \psi_{3}\right)$ is given by $\Pi z=\left\langle z, \phi_{1}\right\rangle \psi_{1}+\left\langle z, \phi_{2}\right\rangle \psi_{2}+\left\langle z, \phi_{3}\right\rangle \psi_{3}$ with

$$
\begin{gathered}
\phi_{1}=\frac{3}{10 r_{6}\left(r_{2}^{2}-r_{5}^{2}\right)}\left(\begin{array}{c}
10 r_{6}\left(r_{2}^{2}-r_{5}^{2}\right) x \\
0 \\
0 \\
5 r_{5} r_{6} x^{2}+r_{2}^{2}-r_{5}^{2}-2 r_{5} r_{6}
\end{array}\right), \\
\phi_{2}=\frac{3 r_{2}}{r_{2}^{2}-r_{5}^{2}}\left(\begin{array}{l}
0 \\
0 \\
x \\
0
\end{array}\right), \phi_{3}=\frac{3 r_{1} r_{2}}{r_{6}\left(r_{2}^{2}-r_{5}^{2}\right)}\left(\begin{array}{l}
0 \\
0 \\
0 \\
1
\end{array}\right),
\end{gathered}
$$

and $\left\langle z, \phi_{i}\right\rangle:=\int_{0}^{1} z(x) \phi_{i}(x) \mathrm{d} x$. It commutes with $\mathcal{L}$ and with $\mathcal{R}$ given by (14): $\Pi \mathcal{L}=\mathcal{L} \Pi$ and $\Pi \mathcal{R}=\mathcal{R} \Pi$. Moreover $\mathcal{R} \psi_{1}=\psi_{1}, \mathcal{R} \psi_{2}=-\psi_{2}$ and $\mathcal{R} \psi_{3}=\psi_{3}$.

Let us still denote the compression force (3) seen as a function of $z$ by $P$. Then $P=P(z)$ is a smooth function of $z$ for the $Z$ topology and for $z$ in some neighborhood of the origin in $D(\mathcal{L})$. Moreover tedious computation gives

$$
P(z)=\mathcal{P}\left(\beta_{0}\right)-\frac{r_{6}\left(r_{2}^{2}-r_{5}^{2}\right)}{12 r_{1} r_{2}} \xi_{3}+O\left(|z|_{Z}^{2}\right) .
$$




\section{Reduction to a Center Manifold}

We can apply the center-manifold theorem [15] (without parameter; see also Theorem 2.1 in [14]): problem (15) has a center manifold

$$
M=\left\{z \in D(\mathcal{L}): z=\sum_{i=1}^{3} \xi_{i} \psi_{1}+h(\xi),|\xi|<<1\right\} .
$$

where $\xi=\left(\xi_{1}, \xi_{2}, \xi_{3}\right) \in \mathbb{R}^{3}$ and $h(\xi)=O\left(|\xi|^{2}\right)$ as $\xi \rightarrow 0$. For simplicity, we shall identify $\sum_{i=1}^{3} \xi_{i} \psi_{i} \in \Pi Z$ with $\xi \in \mathbb{R}^{3}$. By definition of a center manifold, for any fixed $k \in \mathbb{N}$ there exists a neighborhood $U \subset D(\mathcal{L})$ of the origin such that

1. $h \in C^{k}(U \cap \Pi Z, D(\mathcal{L}) \cap((1-\Pi) Z))(U$ might depend on $k)$,

2. $M$ is locally invariant, that is, through every point in $M$ there is a solution that stays in $M$ as long as it remains in $U$,

3. every solution $z: \mathbb{R} \rightarrow D(\mathcal{L})$ (defined for all $t$ !) that stays in $U$ lies completely in $M$,

4. every solution $\tilde{z}_{1}:\left(t_{1}, t_{2}\right) \rightarrow U \cap(\Pi Z)$ of the reduced equation in $\Pi Z$

$$
\dot{z}_{1}=\mathcal{L} z_{1}+\Pi f\left(z_{1}+h\left(z_{1}\right)\right)
$$

leads to the solution $\widetilde{z}(t):=\widetilde{z}_{1}(t)+h\left(\widetilde{z}_{1}(t)\right), t \in\left(t_{1}, t_{2}\right)$,

5. the reduced equation is reversible with respect to $\left.\mathcal{R}\right|_{\Pi Z}: \Pi Z \rightarrow \Pi Z$,

6. the compression function $U \cap \Pi Z \ni z \rightarrow P(z+h(z)) \in \mathbb{R}$ is constant along orbits of the reduced equation.

The identification of $\Pi Z$ with $\mathbb{R}^{3}$ leads to the reversibility

$$
\left(\xi_{1}, \xi_{2}, \xi_{3}\right) \rightarrow\left(\xi_{1},-\xi_{2}, \xi_{3}\right)
$$

The reduced equation is

$$
\left\{\begin{array}{l}
\dot{\xi}_{1}=\xi_{2}+a_{1} \xi_{1} \xi_{2}+a_{2} \xi_{2} \xi_{3}+c_{1} \xi_{1} \xi_{3}+c_{2} \xi_{1}^{2}+c_{3} \xi_{2}^{2}+c_{4} \xi_{3}^{2}+O\left(|\xi|^{3}\right), \\
\dot{\xi}_{2}=\xi_{3}+a_{3} \xi_{1} \xi_{3}+a_{4} \xi_{2}^{2}+a_{5} \xi_{3}^{2}+c_{5} \xi_{1} \xi_{2}+c_{6} \xi_{2} \xi_{3}+d \xi_{1}^{2}+O\left(|\xi|^{3}\right), \\
\dot{\xi}_{3}=a_{6} \xi_{1} \xi_{2}+a_{7} \xi_{2} \xi_{3}+c_{7} \xi_{1} \xi_{3}+c_{8} \xi_{1}^{2}+c_{9} \xi_{2}^{2}+c_{10} \xi_{3}^{2}+O\left(|\xi|^{3}\right),
\end{array}\right.
$$

By reversibility, $c_{1}=\ldots=c_{10}=0$. Moreover $d=0$ (the computation of $d$ and $a_{6}$ below is tedious). Among the remaining constants, only the value of $a_{6}$ is relevant to what follows:

$$
a_{6}=\frac{3 r_{1} r_{2} r_{6}}{\left(r_{2}^{2}-r_{5}^{2}\right) r_{4}^{2}} \mathcal{P}^{\prime \prime}\left(\beta_{0}\right)
$$

where $\mathcal{P}^{\prime \prime}\left(\beta_{0}\right)$ is given in (7). Note that $a_{6}$ has the same sign as $\mathcal{P}^{\prime \prime}\left(\beta_{0}\right)$. 


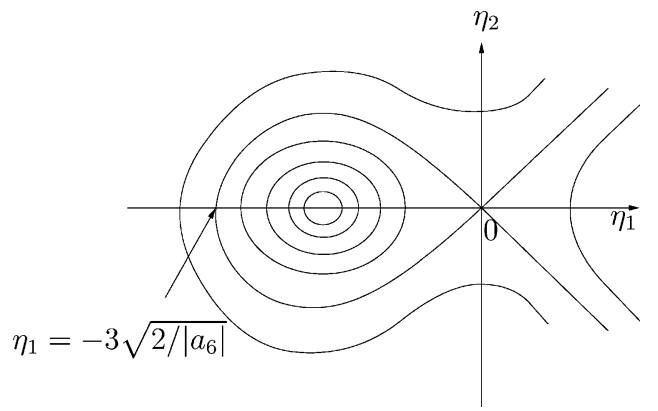

Figure 3. Level sets of $\eta_{2}^{2}-\sqrt{2\left|a_{6}\right|} \eta_{1}^{2}-\left|a_{6} / 3\right| \eta_{1}^{3}$.

The compression force is given by $P=P(\xi)=\mathcal{P}\left(\beta_{0}\right)-\frac{r_{6}\left(r_{2}^{2}-r_{5}^{2}\right)}{12 r_{1} r_{2}} \xi_{3}+O\left(|\xi|^{2}\right)$ (see (18)). As $P$ does not depend on $t$,

$$
\sigma:=\frac{12 r_{1} r_{2}}{r_{6}\left(r_{2}^{2}-r_{5}^{2}\right)}\left(P-\mathcal{P}\left(\beta_{0}\right)\right)=-\xi_{3}+O\left(|\xi|^{3}\right),
$$

is an invariant of (19) (its sign is the same as the one of $\mathcal{P}^{\prime \prime}\left(\beta_{0}\right)$. As a consequence

$$
\sigma=-\xi_{3}+\frac{a_{6}}{2} \xi_{1}^{2}+b_{2} \xi_{2}^{2}+b_{3} \xi_{3}^{2}+\left(a_{7}-2 b_{2}\right) \xi_{1} \xi_{3}+O\left(|\xi|^{3}\right)
$$

where $b_{2}, b_{3}$ are constants. System (19) is then reduced to

$$
\left\{\begin{aligned}
\dot{\xi}_{1}= & \xi_{2}+a_{1} \xi_{1} \xi_{2}-a_{2} \sigma \xi_{2}+O\left(\left|\xi_{1}, \xi_{2}, \sigma\right|^{3}\right) \\
\dot{\xi}_{2}= & -\sigma+\frac{a_{6}}{2} \xi_{1}^{2}+\left(a_{4}+b_{2}\right) \xi_{2}^{2}+\left(a_{5}+b_{3}\right) \sigma^{2}-\left(a_{3}+a_{7}-2 b_{2}\right) \sigma \xi_{1} \\
& +O\left(\left|\xi_{1}, \xi_{2}, \sigma\right|^{3}\right) .
\end{aligned}\right.
$$

If $\sigma / a_{6}>0$, there is an equilibrium of the form $\left(\bar{\xi}_{1}, \bar{\xi}_{2}\right)=\left(\operatorname{sgn}\left(a_{6}\right) \sqrt{2 \sigma / a_{6}}, 0\right)$ $+O\left(|\sigma|^{3 / 2}\right)$. By reversibility, $\bar{\xi}_{2}=0$. Equation (22) can be written in terms of $\tilde{\xi}_{1}:=\xi_{1}=\bar{\xi}_{1}$ and $\tilde{\xi}_{2}:=\xi_{2}-\bar{\xi}_{2}=\xi_{2}$ as

$$
\left\{\begin{array}{l}
\dot{\tilde{\xi}}_{1}=\tilde{\xi}_{2}+a_{1} \tilde{\xi}_{1} \tilde{\xi}_{2}+O\left(\left|\tilde{\xi}_{1}, \tilde{\xi}_{2}\right|^{3}\right)+|\sigma|^{1 / 2} O\left(\left|\left(\tilde{\xi}_{1}, \tilde{\xi}_{2}\right)\right|\right), \\
\dot{\tilde{\xi}}_{2}=\left|a_{6}\right| \sqrt{2 \sigma / a_{6}} \tilde{\xi}_{1}+\frac{a_{6}}{2} \tilde{\xi}_{1}^{2}+\left(a_{4}+b_{2}\right) \tilde{\xi}_{2}^{2}+O\left(\left|\left(\tilde{\xi}_{1}, \tilde{\xi}_{2}\right)\right|^{3}\right)+|\sigma| O\left(\left|\left(\tilde{\xi}_{1}, \tilde{\xi}_{2}\right)\right|\right) .
\end{array}\right.
$$

For $0<\epsilon<<1$, we now set $\sigma=\operatorname{sgn}\left(a_{6}\right) \epsilon^{4}, s=\epsilon t, \tilde{\xi}_{1}(t)=\operatorname{sgn}\left(a_{6}\right) \epsilon^{2} \eta_{1}(s)$ and 
$\tilde{\xi}_{2}(t)=\operatorname{sgn}\left(a_{6}\right) \epsilon^{3} \eta_{2}(s)$, so that $(22)$ is transformed into

$$
\left\{\begin{array}{l}
\eta_{1}^{\prime}=\eta_{2}+\epsilon^{2} O\left(\left|\left(\eta_{1}, \eta_{2}\right)\right|\right), \\
\eta_{2}^{\prime}=\sqrt{2\left|a_{6}\right|} \eta_{1}+\frac{\left|a_{6}\right|}{2} \eta_{1}^{2}+\epsilon^{2} O\left(\left|\left(\eta_{1}, \eta_{2}\right)\right|\right),
\end{array}\right.
$$

where ${ }^{\prime}=\frac{d}{d s}$. The problem is now reversible under $\left(\eta_{1}, \eta_{2}\right) \rightarrow\left(\eta_{1},-\eta_{2}\right)$. Note also that (23) is well-defined for negative $\epsilon$ near zero and that the right-hand side can be assumed as (finitely) many times differentiable with respect to $\left(\eta_{1}, \eta_{2}, \epsilon\right)$ as needed.

For all small $|\epsilon|$, the origin is a hyperbolic equilibrium of (23). Moreover, when $\epsilon=0, \eta_{2}^{2}-\sqrt{2\left|a_{6}\right|} \eta_{1}^{2}-\left|a_{6} / 3\right| \eta_{1}^{3}$ is a preserved quantity. The set

$$
\left\{\left(\eta_{1}, \eta_{2}\right) \in \mathbb{R}^{2}: \eta_{1} \leq 0, \eta_{2}^{2}-\sqrt{2\left|a_{6}\right|} \eta_{1}^{2}-\left|a_{6} / 3\right| \eta_{1}^{3}=0\right\}
$$

is a closed curve that contains the origin (the only singular point of the curve) and that crosses the semi-axis $(-\infty, 0) \times\{0\}$ transversally at $\eta_{1}=-3 \sqrt{2 /\left|a_{6}\right|}$ (see Figure 3). In other words, equation (19) has a homoclinic orbit to the origin that crosses transversally the semi-axis $(-\infty, 0) \times\{0\}$, that is, the onedimensional global unstable manifold crosses transversally the semi-axis $(-\infty, 0)$ $\times\{0\}$. As the local unstable manifold depends regularly on $\epsilon$, the onedimensional global unstable manifold still crosses transversally this semi-axis for all small $\epsilon>0$. The semi-axis being invariant under the reversibility $\left(\eta_{1}, \eta_{2}\right) \rightarrow$ $\left(\eta_{1},-\eta_{2}\right)$, problem (23) has a homoclinic orbit for all small $\epsilon>0$. Going back to (1) and (2), they correspond to localized deformations of the elastic band.

\section{Appendix A}

Proof of Lemma 2, Part 1. This appendix establishes the first part of Lemma 2. We assume $0 \neq s \in \mathbb{R}$, and have to show that the equation $(\mathcal{L}-i s) z=0$ only admits the trivial solution in $D(\mathcal{L})$. Introducing the independent variable $\xi=s x$, setting $\tilde{u}_{2}=u_{2}+\left(\left\langle p_{2}\right\rangle-r_{6} u_{1}(1)\right) /\left(i s r_{4}\right)$ and eliminating $p_{1}, p_{2}$, the problem becomes

$$
\left\{\begin{array}{l}
r_{1} u_{1}^{\prime \prime}+i\left(r_{5}+r_{6}\right) \tilde{u}_{2}^{\prime}-r_{2} u_{1}=0, \\
r_{2} \tilde{u}_{2}^{\prime \prime}+i\left(r_{5}+r_{6}\right) \tilde{u}_{1}^{\prime}-r_{4} \tilde{u}_{2}=0, \\
u_{1}=0 \text { and } \tilde{u}_{2}^{\prime}=0 \text { when } \xi=0 \\
i r_{6} \tilde{u}_{2}+r_{1} u_{1}^{\prime}=0 \text { and } i r_{5} u_{1}+r_{2} \tilde{u}_{2}^{\prime}=0 \text { when } \xi=s,
\end{array}\right.
$$

where ' denotes $d / \mathrm{d} \xi$. Defining the parameters

$$
R_{1}=\frac{r_{1}}{r_{2}}, \quad R_{5}=\frac{r_{5}}{r_{2}}, \quad R=\frac{r_{5}+2 r_{6}}{r_{2}},
$$


which satisfy the inequalities $R_{1}>0, R<1<R_{5}$, the problem reads

$$
\left\{\begin{array}{l}
2 R_{1} u_{1}^{\prime \prime}+i\left(R+R_{5}\right) \tilde{u}_{2}^{\prime}-2 u_{1}=0, \\
4 R_{1} \tilde{u}_{2}^{\prime \prime}+2 i R_{i}\left(R+R_{5}\right) u_{1}^{\prime}-\left(R_{5}-R\right)^{2} \tilde{u}_{2}=0, \\
u_{1}=0 \text { and } \tilde{u}_{2}^{\prime}=0 \text { when } \xi=0, \\
i\left(R_{5}-R\right) \tilde{u}_{2}+2 R_{1} u_{1}^{\prime}=0 \text { and } i R_{5} u_{1}+\tilde{u}_{2}^{\prime}=0 \text { when } \xi=s .
\end{array}\right.
$$

Assuming $\left(u_{1}, \tilde{u}_{2}\right)$ is a solution, then $\left(u_{1}+\overline{u_{1}}, u_{2}-\overline{\tilde{u}_{2}}\right)$ is also a solution. Thus if the problem admits a nontrivial solution, then it also admits a nontrivial solution of the form $\left(u_{1}, \tilde{u}_{2}\right)=\left(w_{1}, i w_{2}\right)$, where $w_{1}, w_{2}$ are real functions. It is therefore sufficient to show that the problem

$$
\left\{\begin{array}{l}
2 R_{1} w_{1}^{\prime \prime}+i\left(R+R_{5}\right) w_{2}^{\prime}-2 w_{1}=0, \\
4 R_{1} w_{2}^{\prime \prime}+2 R_{i}\left(R+R_{5}\right) w_{1}^{\prime}-\left(R_{5}-R\right)^{2} w_{2}=0, \\
w_{1}=0 \text { and } w_{2}^{\prime}=0 \text { when } \xi=0, \\
\left(R-R_{5}\right) w_{2}+2 R_{1} w_{1}^{\prime}=0 \text { and } R_{5} w_{1}+w_{2}^{\prime}=0 \text { when } \xi=s
\end{array}\right.
$$

does not admit any nontrivial real solution. Before solving this problem it is useful to remark the following. Assume $\left(w_{1}, w_{2}\right)$ is a solution to the differential equations that satisfies the boundary conditions at $\xi=0$. Then $\widehat{w}_{1}(\xi)=$ $w_{1}(\xi)+w_{1}(-\xi), \widehat{w}_{2}(\xi)=w_{2}(\xi)-w_{2}(-\xi)$ also constitutes a solution to the differential equations. Moreover $\widehat{w}_{1}(0)=\widehat{w}^{\prime}{ }_{1}(0)=0$ and $\widehat{w}_{2}(0)=\widehat{w}_{2}^{\prime}(0)=0$, which implies $\widehat{w}_{1}(\xi)=\widehat{w}_{2}(\xi)=0, \forall \xi$. Thus any solution to the differential equations which satisfies the boundary conditions at $\xi=0$ possesses the parity properties $w_{1}(-\xi)=-w_{1}(\xi)$ and $w_{2}(-\xi)=w_{2}(\xi)$. Note moreover that, defining $\gamma(\xi)=R_{5} w_{1}(\xi)+w_{2}^{\prime}(\xi)$, the boundary conditions at $\xi=s$ can be written as $\gamma(s)=$ $\gamma^{\prime}(s)=0$. For solving problem (26) we distinguish four cases:

1. Assume $R<-1$ and $R \neq-R 5$. Defining the real quantities

$$
\Delta=\sqrt{\left(R^{2}-1\right)\left(R_{5}^{2}-1\right)}, \quad \rho_{1,2}=\sqrt{\frac{1-R R_{5} \mp \Delta}{2 R_{1}}},
$$

the solutions to the differential equations which satisfy the boundary conditions at $\xi=0$ are of the form

$$
\begin{aligned}
& w_{1}(\xi)=a \rho_{1}\left(R+R_{5}\right) \sinh \rho_{1} \xi+b \rho_{2}\left(R+R_{5}\right) \sinh \rho_{2} \xi \\
& w_{2}(\xi)=-a\left(1+R R_{5}+\Delta\right) \cosh \rho_{1} \xi-b\left(1+R R_{5}-\Delta\right) \cosh \rho_{2} \xi
\end{aligned}
$$

with $a, b \in \mathbb{R}$. The boundary conditions at $\xi=s$ become

$$
\begin{aligned}
\gamma(s) & =a \rho_{1}\left(R_{5}^{2}-1-\Delta\right) \sinh \rho_{1} s+b \rho_{2}\left(R_{5}^{2}-1+\Delta\right) \sinh \rho_{2} s=0, \\
\gamma^{\prime}(s) & =a \rho_{1}^{2}\left(R_{5}^{2}-1-\Delta\right) \cosh \rho_{1} s+b \rho_{2}^{2}\left(R_{5}^{2}-1+\Delta\right) \cosh \rho_{2} s=0 .
\end{aligned}
$$


This system admits nontrivial solutions for $\mathrm{a}, \mathrm{b}$ only if $\rho_{1} \tanh \rho_{2} \mathrm{~s}=\rho_{2} \tanh$ $\rho_{1} s$, which is impossible since $s \neq 0$ and $\rho_{2}>\rho_{1}$. Indeed, setting $\sigma=\rho_{1} s$, $\widehat{\rho}=\rho_{2} / \rho_{1}$ and $\varphi(\sigma)=\tanh \widehat{\rho} \sigma-\widehat{\rho} \tanh \sigma$, the equality can be rewritten as $\varphi(\sigma)=0$. We have $\varphi(0)=0, \varphi^{\prime}(\sigma)>0$ when $\sigma<0$ and $\varphi^{\prime}(\sigma)<0$ when $\sigma>$ 0 . In our case $\sigma \neq 0$ and therefore $\varphi(\sigma) \neq 0$. We conclude that the problem only admits the trivial solution.

2. Assume $R>-1$. Define the real quantities

$$
\Delta=\sqrt{\left(1-R^{2}\right)\left(R_{5}^{2}-1\right)}, \quad \Omega=1-R R_{5},
$$

and let $\alpha+i \beta$ be that square root of $(\Omega+i \Delta) /\left(2 R^{1}\right)$ which lies in the first quadrant, i.e., with $\alpha, \beta>0$. Note the relations $2 R_{1}\left(\alpha^{2}-\beta^{2}\right)=\Omega, 4 R_{1} \alpha \beta=$ $\Delta$ and $2 R_{1}\left(\alpha^{2}+\beta^{2}\right)=\left(R_{5}-R\right)$. The solutions to the differential equations which satisfy the boundary conditions at $\xi=0$ take the form

$$
\begin{aligned}
w_{1}(\xi)= & \left(R_{5}^{2}-R^{2}\right)[a \sinh \alpha \xi \cos \beta \xi+b \cosh \alpha \xi \sin \beta \xi], \\
w_{2}(\xi)= & -2 R_{1}\left[\left(R_{5}-R+2\right) \beta a-\left(R_{5}-R-2\right) \alpha b\right] \sinh \alpha \xi \sin \beta \xi \\
& +2 R_{1}\left[\left(R_{5}-R-2\right) \alpha a+\left(R_{5}-R+2\right) \beta b\right] \cosh \alpha \xi \cos \beta \xi,
\end{aligned}
$$

with $a, b \in \mathbb{R}$. Introducing the quantities

$$
\left(\begin{array}{l}
\tilde{a} \\
\tilde{b}
\end{array}\right)=\left(R_{5}-R\right)\left(\begin{array}{cc}
R_{5}^{2}-1 & \Delta \\
-\Delta & R_{5}^{2}-1
\end{array}\right)\left(\begin{array}{l}
a \\
b
\end{array}\right)
$$

the boundary conditions at $\xi=s$ become

$$
\begin{aligned}
\gamma(s)= & \tilde{a} \sinh \alpha s \cos \beta s+\tilde{b} \cosh \alpha s \sin \beta s=0, \\
\gamma^{\prime}(s)= & \tilde{a}(\alpha \cosh \alpha s \cos \beta s-\beta \sinh \alpha s \sin \beta s) \\
& +\tilde{b}(\alpha \sinh \alpha s \sin \beta s+\beta \cosh \alpha s \cos \beta s)=0 .
\end{aligned}
$$

This system admits nontrivial solutions for $\widetilde{a}, \widetilde{b}$ only if $\alpha \sin 2 \beta \mathrm{s}=\beta \sinh$ $2 \alpha \mathrm{s}$, which is impossible since $s \neq 0$ and $\alpha, \beta>0$. Therefore $(\widetilde{a}, \widetilde{b})=0$ and, since the matrix appearing in (31) is regular, $(a, b)=0$. We conclude that the problem only admits the trivial solution.

3. The third case $R=-R_{5}$ and the last case $R=-1$ are dealt with in an analogous manner.

\section{Appendix B}

Proof of Lemma 2, Part 2. In Appendix A it was shown that $\mathcal{L}-$ is is injective for all $s \neq 0$. Assuming $|s|>s_{0}$, where $s_{0}>0$ is chosen large enough, we now prove that for any $(f, g)=\left(f_{1}, f_{2}, g_{1}, g_{2}\right) \in Z$, the equation $(\mathcal{L}-i s) z=(f, g)$ 
admits a solution $z \in D(\mathcal{L})$. Note that the solution is unique by injectivity. We also establish the estimate

$$
|s|\|z\|_{Z} \leq \text { Const }\|(f, g)\| z,
$$

where the constant depends neither on $|s|>s_{0}$ nor on $(f, g)$. Setting

$$
\tilde{u}_{1}=u_{1}, \quad \tilde{u}_{2}=u_{2}+\frac{\left\langle p_{2}\right\rangle-r_{6} u_{1}(1)}{i s r_{4}}, \quad v_{1}=\frac{p_{1}-r_{5} u_{2}^{\prime}}{r_{2}}, \quad v_{2}=\frac{p_{2}-r_{6} u_{1}^{\prime}}{r_{4}},
$$

and $\widetilde{g}_{1}=g_{1}-r_{5} f_{2}^{\prime}, \widetilde{g}_{2}=g_{2}-r_{6} f_{1}^{\prime}$, the problem becomes

$$
\left\{\begin{array}{l}
v_{1}=i s \tilde{u}_{1}+f_{1}, \\
v_{2}=i s \tilde{u}_{2}+f_{2}, \\
-r_{1} \tilde{u}_{1}^{\prime \prime}-r_{6} v_{2}^{\prime}-i s r_{5} \tilde{u}_{2}^{\prime}-i s r_{2} v_{1}=\tilde{g}_{1}+r_{5} f_{2}^{\prime}, \\
-r_{2} \tilde{u}_{2}^{\prime \prime}-r_{5} v_{1}^{\prime}-i s r_{6} \tilde{u}_{1}^{\prime}-i s r_{4} v_{2}=\tilde{g}_{2}+r_{6} f_{1}^{\prime}, \\
\tilde{u}_{1}=0 \text { and } r_{5} v_{1}+r_{2} \tilde{u}_{2}^{\prime}=0 \text { at } x=0, \\
r_{6} v_{2}+r_{1} \tilde{u}_{1}^{\prime}=0 \text { and } r_{5} v_{1}+r_{2} \tilde{u}_{2}^{\prime}=0 \text { at } x=1 .
\end{array}\right.
$$

We have to show the existence of a solution $(\tilde{u}, v)=\left(\tilde{u}_{1}, \tilde{u}_{2}, v_{1}, v_{2}\right) \in W^{2,2}(0,1)$ $\times W^{2,2}(0,1) \times W^{1,2}(0,1) \times W^{1,2}(0,1)$ and establish that

$$
\|\tilde{u}\|_{W^{1,2}(0,1)}+\|v\|_{L^{2}(0,1)} \leq \text { Const }|s|^{-1}\left\{\|f\|_{W^{1,2}(0,1)}+\|\tilde{g}\|_{L^{2}(0,1)}\right\} .
$$

By linearity, we can proceed in two steps: first assume $f=0$ and then $\widetilde{g}=0$. Step 1: Assume $f=0$. In this case $\widetilde{g}=g$. Eliminating $v_{1}$ and $v_{2}$, the problem becomes

$$
\left\{\begin{array}{l}
-r_{1} \tilde{u}_{1}^{\prime \prime}-\left(r_{5}+r_{6}\right) i s \tilde{u}_{2}^{\prime}+r_{2} s^{2} \tilde{u}_{1}=g_{1}, \\
-r_{2} \tilde{u}_{2}^{\prime \prime}-\left(r_{5}+r_{6}\right) i s \tilde{u}_{1}^{\prime}+r_{4} s^{2} \tilde{u}_{2}=g_{2}, \\
\tilde{u}_{1}=0 \text { and } \tilde{u}_{2}^{\prime}=0 \text { at } x=0, \\
r_{6} i s \tilde{u}_{2}+r_{1} \tilde{u}_{1}^{\prime}=0 \text { and } r_{5} i s \tilde{u}_{1}+r_{2} \tilde{u}_{2}^{\prime}=0 \text { at } x=1,
\end{array}\right.
$$

We have to prove the existence of a solution $\tilde{u} \in W^{2,2}(0,1) \times W^{2,2}(0,1)$ and show that

$$
\|\tilde{u}\|_{W^{1,2}(0,1)}+|s|\|\tilde{u}\|_{L^{2}(0,1)} \leq \text { Const }|s|^{-1}\left\{\|g\|_{L^{2}(0,1)}+\|\tilde{u}\|_{L^{2}(0,1)}\right\} .
$$

This problem is self-adjoint and compact, and therefore existence is a consequence of uniqueness. The issue is to check the estimate on the norms. In 
this order, for a solution $\tilde{u}$, we set $\mu(x, y)=e^{i s y} \tilde{u}(x)$ and observe that $\mu \in$ $W^{2,2}((0,1) \times(0, P)) \times W^{2,2}((0,1) \times(0, P))$, with $P=2 k \pi / s \in[1,2]$ and $k \in \mathbb{N}$. Moreover

$$
\left\{\begin{array}{l}
-r_{1} \partial_{1}^{2} \mu_{1}-\left(r_{5}+r_{6}\right) \partial_{12}^{2} \mu_{2}-r_{2} \partial_{2}^{2} \mu_{1}=g_{1} e^{i s y} \\
-r_{2} \partial_{1}^{2} \mu_{2}-\left(r_{5}+r_{6}\right) \partial_{12}^{2} \mu_{1}-r_{4} \partial_{2}^{2} \mu_{2}=g_{2} e^{i s y} \\
\mu_{1}=0 \text { and } \partial_{1} \mu_{2}=0 \text { when } x=0 \\
r_{6} \partial_{2} \mu_{2}+r_{1} \partial_{1} \mu_{1}=0 \text { and } r_{5} \partial_{2} \mu_{1}+r_{2} \partial_{1} \mu_{2}=0 \text { when } x=1, \\
\mu_{1}(x, P)=\mu_{1}(x, 0), \partial_{2} \mu_{1}(x, P)=\partial_{2} \mu_{1}(x, 0) \text { for all } x \in(0,1), \\
\mu_{2}(x, P)=\mu_{2}(x, 0), \partial_{2} \mu_{2}(x, P)=\partial_{2} \mu_{2}(x, 0) \text { for all } x \in(0,1) .
\end{array}\right.
$$

Hence it suffices to show

$$
\|\mu\|_{W^{2,2}((0,1) \times(0, P))} \leq \text { Const }\left\{\|g\|_{L^{2}(0,1)}+\|\mu\|_{L^{2}((0,1) \times(0, P))}\right\} .
$$

In fact, according to proposition 9.1 in Simpson and Spector [19], this inequality holds since problem (34) is strongly elliptic and satisfies the complementing condition. We proceed by checking this last condition, which means that if we consider the equations

$$
\left\{\begin{array}{l}
r_{1} u_{1}^{\prime \prime}+\left(r_{5}+r_{6}\right) i s u_{2}^{\prime}-r_{2} s^{2} u_{1}=0 \\
r_{2} u_{2}^{\prime \prime}+\left(r_{5}+r_{6}\right) i s u_{1}^{\prime}-r_{4} s^{2} u_{2}=0
\end{array}\right.
$$

then $u=0$ is the only bounded solution on $[0, \infty)$ such that $u_{1}(0)=u_{2}^{\prime}(0)=0$, and $u=0$ is the only bounded solution on $(-\infty, 0]$ such that $r_{6} i s u_{2}(0)+r_{1} u_{1}^{\prime}(0)=$ $r_{5} i s u_{1}(0)+r_{2} u_{2}^{\prime}(0)=0$ (cf. [19], Section 4). Introducing the independent variable $\xi=s x$ and the parameters $R_{1}, R_{5}, R$ defined in equation (24), the problem can be written as

$$
\left\{\begin{array}{l}
2 R_{1} u_{1}^{\prime \prime}+i\left(R+R_{5}\right) u_{2}^{\prime}-2 u_{1}=0, \\
4 R_{1} u_{2}^{\prime \prime}+2 i R_{1}\left(R+R_{5}\right) u_{1}^{\prime}-\left(R_{5}-R\right)^{2} u_{2}=0, \\
u_{1}(0)=u_{2}^{\prime}(0)=0 \text { or } i\left(R-R_{5}\right) u_{2}(0)+2 R_{1} u_{1}^{\prime}(0)=i R_{5} u_{1}(0)+u_{2}^{\prime}(0)=0
\end{array}\right.
$$

(compare with (25)). If $\left(u_{1}, u_{2}\right)$ is a bounded solution, then $\left(u_{1}+\overline{u_{1}}, u_{2}-\overline{u_{2}}\right)$ is also a bounded solution. Thus if the problem admits a nontrivial bounded solution, then it also admits a nontrivial bounded solution of the form $\left(u_{1}, u_{2}\right)=$ $\left(w_{1}, i w_{2}\right)$, where $w_{1}, w_{2}$ are real functions. To show that the problem satisfies the complementing condition it is therefore sufficient to prove that $w=0$ is the only bounded solution to

$$
\left\{\begin{array}{l}
2 R_{1} w_{1}^{\prime \prime}+\left(R+R_{5}\right) w_{2}^{\prime}-2 w_{1}=0 \\
4 R_{1} w_{2}^{\prime \prime}+2 R_{1}\left(R+R_{5}\right) w_{1}^{\prime}-\left(R_{5}-R\right)^{2} w_{2}=0 \\
w_{1}(0)=w_{2}^{\prime}(0)=0 \text { or }\left(R-R_{5}\right) w_{2}(0)-2 R_{1} w_{1}^{\prime}(0)=R_{5} w_{1}^{\prime}(0)+w_{2}^{\prime}(0)=0
\end{array}\right.
$$


(compare with (26)). Defining $\gamma(\xi)=R_{5} w_{1}(\xi)+w_{2}^{\prime}(\xi)$, the second pair of boundary conditions can be written as $\gamma(0)=\gamma^{\prime}(0)=0$. We now distinguish four cases:

1. Assume $R<-1$ and $R \neq-R 5$, and define $\Delta, \rho_{1}, \rho_{2}$ as in (27). The solutions on $[0, \infty)$ to the differential equations satisfying the first pair of boundary conditions are expressed in (28). Among these, only the trivial solution is bounded. The bounded solutions on $(-\infty, 0]$ to the differential equations are

$$
\begin{aligned}
& w_{1}(\xi)=a \rho_{1}\left(R+R_{5}\right) e^{\rho_{1} \xi}+b \rho_{2}\left(R+R_{5}\right) e^{\rho_{2} \xi}, \\
& w_{2}(\xi)=-a\left(1+R R_{5}+\Delta\right) e^{\rho_{1} \xi}-b\left(1+R R_{5}-\Delta\right) e^{\rho_{2} \xi} .
\end{aligned}
$$

The second set of boundary conditions then becomes

$$
\begin{aligned}
\gamma(0) & =a \rho_{1}\left(-1+R_{5}^{2}-\Delta\right)+b \rho_{2}\left(-1+R_{5}^{2}+\Delta\right)=0, \\
\gamma^{\prime}(0) & =a \rho_{1}^{2}\left(-1+R_{5}^{2}-\Delta\right)+b \rho_{2}^{2}\left(-1+R_{5}^{2}+\Delta\right)=0 .
\end{aligned}
$$

which implies $a=b=0$.

2. Assume $R>-1$, and consider the numbers $\alpha$ and $\beta$ defined below equation (29). The solutions on $[0, \infty)$ to the differential equations satisfying the first pair of boundary conditions are expressed in equation (30). Among these, only the trivial solution is bounded. The bounded solutions on $(-\infty, 0]$ to the differential equations are

$$
\begin{aligned}
w_{1}(\xi)= & \left(R_{5}^{2}-R^{2}(a \cos \beta \xi+b \sin \beta \xi)\right) e^{\alpha \xi}, \\
w_{2}(\xi)= & 2 R_{1}\left[\left(R_{5}-R-2\right) \alpha a+\left(R_{5}-R+2\right) \beta b\right] \cos \beta \xi e^{\alpha \xi} \\
& +2 R_{1}\left[-\left(R_{5}-R+2\right) \beta a+\left(R_{5}-R-2\right) \alpha b\right] \sin \beta \xi e^{\alpha \xi} .
\end{aligned}
$$

The second set of boundary conditions then becomes

$$
\begin{aligned}
\gamma(0) & =\left(R_{5}-R\right)\left[\left(R_{5}^{2}-1\right) a+4 R_{1} \alpha \beta b\right]=0, \\
\gamma^{\prime}(0) & =\left(R_{5}-R\right)^{2}\left[\left(R_{5}-1\right) \alpha a+\left(R_{5}+1\right) \beta b\right]=0 .
\end{aligned}
$$

The inequality $4 R_{1} \alpha^{2}=\left(R_{5}+1\right)(1-R) \neq(\mathrm{R} 5+1)^{2}$ implies that the determinant associated with the linear system for $\mathrm{a}, \mathrm{b}$ is nonzero, and thus $a=b=0$.

3. The third case $R=-R 5$ and the last case $R=-1$ are dealt with in an analogous manner.

Step 2: Assume $\widetilde{g}=0$. We restrict the following analysis to $f \in W^{2,2}(0,1) \times$ $W^{2,2}(0,1)$ satisfying $f_{1}(0)=0$ and $\left\langle f_{2}\right\rangle=0$. Since the set of functions $f$ considered is dense in the set of all possible functions $f$, the results stay valid in the general case. Eliminating $\tilde{u}_{1}$ and $\tilde{u}_{2}$ from (33), we obtain 


$$
\left\{\begin{array}{l}
-r_{1}\left(v_{1}-f_{1}\right)^{\prime \prime}-i s r_{5}\left(v_{2}-f_{2}\right)^{\prime}-i s r_{6} v_{2}^{\prime}+s^{2} r_{2} v_{1}=i s r_{5} f_{2}^{\prime}, \\
-r_{2}\left(v_{2}-f_{2}\right)^{\prime \prime}-i s r_{5} v_{1}^{\prime}-i s r_{6}\left(v_{1}-f_{1}\right)^{\prime}+s^{2} r_{4} v_{2}=i s r_{6} f_{1}^{\prime}, \\
v_{1}=0 \text { and } v_{2}^{\prime}=f_{2}^{\prime} \text { at } x=0 \\
i s r_{6} v_{2}+r_{1} v_{1}^{\prime}=r_{1} f_{1}^{\prime} \text { and } i s r_{5} \mathrm{v}_{1}+r_{2} v_{2}^{\prime}=r_{2} f_{2}^{\prime} \text { at } x=1
\end{array}\right.
$$

We have to prove the existence of a solution $v \in W^{2,2}(0,1) \times W^{2,2}(0,1)$ and show that

$$
\|v\|_{W^{1,2}(0,1)}+|s|\|v\|_{L^{2}(0,1)} \leq \text { Const }\|f\|_{W^{1,2}(0,1)} .
$$

As the problem is self-adjoint, existence for $s \in \mathbb{R} \backslash\{0\}$ is a consequence of uniqueness for the homogeneous problem (that is, $f=0$; see Part 1). It remains to show the above estimate for any solution $v$.

Setting $\eta_{1}(x)=\int_{o}^{x} v_{1} d \xi+A$ and $\eta_{2}(x)=\int_{o}^{x} v_{2} d \xi$ for some constant $A \in \mathbb{C}$ to be given below, we have to show the existence of $\eta \in W^{3,2}(0,1) \times W^{3,2}(0,1)$ and $\kappa_{1}, \kappa_{2} \in \mathbb{C}$ such

$$
\left\{\begin{array}{l}
-r_{1} \eta_{1}^{\prime \prime}-i s\left(r_{5}+r_{6}\right) \eta_{2}^{\prime}+s^{2} r_{2} \eta_{1}=-r_{1} f_{1}^{\prime}+i s \kappa_{1}, \\
-r_{2} \eta_{2}^{\prime \prime}-i s\left(r_{5}+r_{6}\right) \eta_{1}^{\prime}+s^{2} r_{4} \eta_{2}=-r_{2} f_{2}^{\prime}+i s \kappa_{2}, \\
\eta_{1}^{\prime}=0 \text { and } \eta_{2}^{\prime \prime}=f_{2}^{\prime} \text { at } x=0 \\
i s r_{6} \eta_{2}^{\prime}+r_{1} \eta_{1}^{\prime}=r_{1} f_{1}^{\prime} \text { and } i s r_{5} \eta_{1}^{\prime}+r_{2} \eta_{2}^{\prime \prime}=r_{2} f_{2}^{\prime} \text { at } x=1,
\end{array}\right.
$$

that is, such that

$$
\left\{\begin{array}{l}
-r_{1} \eta_{1}^{\prime \prime}-i s\left(r_{5}+r_{6}\right) \eta_{2}^{\prime}+s^{2} r_{2} \eta_{1}=-r_{1} f_{1}^{\prime}+i s \kappa_{1}, \\
-r_{2} \eta_{2}^{\prime \prime}-i s\left(r_{5}+r_{6}\right) \eta_{1}^{\prime}+s^{2} r_{4} \eta_{2}=-r_{2} f_{2}^{\prime}+i s \kappa_{2}, \\
\eta_{1}^{\prime}=0 \text { and } s r_{4} \eta_{2}=i \kappa_{2} \text { at } x=0, \\
r_{5} \eta_{2}^{\prime}+i s r_{2} \eta_{1}^{\prime}=-\kappa_{1} \text { and } r_{6} \eta_{1}^{\prime}+i s r_{4} \eta_{2}=-\kappa_{2} \text { at } x=1 .
\end{array}\right.
$$

Since $\eta_{2}(0)=0$, we have $\kappa_{2}=0$. Setting

$$
A=\frac{-r_{1} f_{1}(1)+i s r_{5} \int_{0}^{1} v_{2} \mathrm{~d} x}{s^{2} r_{2}}-\int_{0}^{1} \int_{0}^{x} v_{1}(\xi) \mathrm{d} \xi \mathrm{d} x=\eta_{1}(0)
$$

implies $\kappa_{1}=0$, and the problem becomes

$$
\left\{\begin{array}{l}
-r_{1} \eta_{1}^{\prime \prime}-i s\left(r_{5}+r_{6}\right) \eta_{2}^{\prime}+s^{2} r_{2} \eta_{1}=-r_{1} f_{1}^{\prime}, \\
-r_{2} \eta_{2}^{\prime \prime}-i s\left(r_{5}+r_{6}\right) \eta_{1}^{\prime}+s^{2} r_{4} \eta_{2}=-r_{2} f_{2}^{\prime}, \\
\eta_{1}^{\prime}=0 \text { and } \eta_{2}=0 \text { at } x=0 \\
r_{5} \eta_{2}^{\prime}+i s r_{2} \eta_{1}=0 \text { and } r_{1} \eta_{1}^{\prime}+i s r_{6} \eta_{2}=0 \text { at } x=1 .
\end{array}\right.
$$

We now have to prove for any solution $\eta \in W^{3,2}(0,1) \times W^{3,2}(0,1)$ the estimate

$$
\left\|\eta^{\prime}\right\|_{W^{1,2}(0,1)}+|s|\|\eta\|_{W^{1,2}(0,1)} \leq \operatorname{Const}\left\{\|f\|_{W^{1,2}(0,1)}+\|\eta\|_{L^{2}(0,1)}\right\} .
$$


In this order we set $\mu(x, y)=e^{i s y} \eta(x)$, where $\eta$ is a solution to equation (38). Observe that $\mu \in W^{2,2}((0,1) \times(0, P)) \times W^{2,2}((0,1) \times(0, P))$, with $P=2 k \pi / s \in$ $[1,2]$ and $k \in \mathbb{N}$.

\section{Moreover}

$$
\left\{\begin{array}{l}
-r_{1} \partial_{1}^{2} \mu_{1}-\left(r_{5}+r_{6}\right) \partial_{12}^{2} \mu_{2}-r_{2} \partial_{2}^{2} \mu_{1}=-r_{1} f_{1}^{\prime} e^{i s y} \\
-r_{2} \partial_{1}^{2} \mu_{2}-\left(r_{5}+r_{6}\right) \partial_{12}^{2} \mu_{1}-r_{4} \partial_{2}^{2} \mu_{2}=-r_{2} f_{2}^{\prime} e^{i s y}, \\
\mu_{2}=0 \text { and } \partial_{1} \mu_{1}=0 \text { when } x=0 \\
r_{6} \partial_{2} \mu_{2}+r_{1} \partial_{1} \mu_{1}=0 \text { and } r_{2} \partial_{2} \mu_{1}+r_{5} \partial_{1} \mu_{2}=0 \text { when } x=1, \\
\mu_{1}(x, P)=\mu_{1}(x, 0), \partial_{2} \mu_{1}(x, P)=\partial_{2} \mu_{1}(x, 0) \text { for all } x \in(0,1), \\
\mu_{2}(x, P)=\mu_{2}(x, 0), \partial_{2} \mu_{2}(x, P)=\partial_{2} \mu_{2}(x, 0) \text { for all } x \in(0,1) .
\end{array}\right.
$$

Hence it suffices to show

$$
\|\mu\|_{W^{2,2}((0,1) \times(0, P))} \leq \mathrm{Const}\left\{\|f\|_{W^{1,2}(0,1)}+\|\mu\|_{L^{2}((0,1) \times(0, P))}\right\} .
$$

In fact, according to proposition 9.1 in Simpson and Spector [19], this inequality holds since problem (39) is strongly elliptic and satisfies the complementing condition. We proceed by checking this last condition, which means that if we consider the equations

$$
\left\{\begin{array}{l}
r_{1} \eta_{1}^{\prime \prime}+i s\left(r_{5}+r_{6}\right) \eta_{2}^{\prime}-s^{2} r_{2} \eta_{1}=0, \\
r_{2} \eta_{2}^{\prime \prime}+i s\left(r_{5}+r_{6}\right) \eta_{1}^{\prime}-s^{2} r_{4} \eta_{2}=0,
\end{array}\right.
$$

then $\eta=0$ is the only bounded solution on $[0, \infty)$ such that $\eta_{1}^{\prime}(0)=\eta_{2}(0)=0$, and $\eta=0$ is the only bounded solution on $(-\infty, 0]$ such that $r_{6} i s \eta_{2}(0)+r_{1} \eta_{1}^{\prime}(0)=$ $r_{2} i s \eta_{1}(0)+r_{5} \eta_{2}^{\prime}(0)=0$ (cf. [19], Section 4). Introducing the independent variable $\xi=s x$ and the parameters $R_{1}, R_{5}, R$ defined in equation (24), the problem can be written as

$$
\left\{\begin{array}{l}
2 R_{1} \eta_{1}^{\prime \prime}+i\left(R+R_{5}\right) \eta_{2}^{\prime}-2 \eta_{1}=0, \\
4 R_{1} \eta_{2}^{\prime \prime}+2 i R_{1}\left(R+R_{5}\right) \eta_{1}^{\prime}-\left(R_{5}-R\right)^{2} \eta_{2}=0, \\
\eta_{1}^{\prime}(0)=\eta_{2}(0)=0 \text { or } i\left(R-R_{5}\right) \eta_{2}(0)+2 R_{1} \eta_{1}^{\prime}(0)=i \eta_{1}(0)+R_{5} \eta_{2}^{\prime}=0 .
\end{array}\right.
$$

If $\left(\eta_{1}, \eta_{2}\right)$ is a bounded solution, then $\left(\eta_{1}+\overline{\eta_{1}}, \eta_{2}-\overline{\eta_{2}}\right)$ is also a bounded solution. Thus if the problem admits a nontrivial bounded solution, then it also admits a nontrivial bounded solution of the form $\left(\eta_{1}, \eta_{2}\right)=\left(w_{1}, i w_{2}\right)$, where $w_{1}$, $w_{2}$ are real functions. To show that the problem satisfies the complementing condition it is therefore sufficient to prove that $w=0$ is the only bounded solution to 


$$
\left\{\begin{array}{l}
2 R_{1} w_{1}^{\prime \prime}-\left(R+R_{5}\right) w_{2}^{\prime}-2 w_{1}=0, \\
4 R_{1} w_{2}^{\prime \prime}+2 R_{1}\left(R+R_{5}\right) w_{1}^{\prime}-\left(R_{5}-R\right)^{2} w_{2}=0, \\
w_{1}^{\prime}(0)=w_{2}(0)=0 \text { or }\left(R-R_{5}\right) w_{2}(0)-2 R_{1} w_{1}^{\prime}(0)=w_{1}(0)+R_{5} w_{2}^{\prime}(0)=0 .
\end{array}\right.
$$

Defining $\gamma(\xi)=R_{5} w_{1}(\xi)+w_{2}^{\prime}(\xi)$, the second pair of boundary conditions can be written as $\gamma^{\prime}(0)=\gamma^{\prime \prime}(0)=0$.

Let $w$ be a bounded solution on $[0, \infty)$ that satisfies the first pair of boundary conditions. Then $w^{\prime}$ is a bounded solution of (35) that satisfies the first pair of boundary conditions of (35). As the trivial solution is the only bounded solution, we get $w^{\prime} \equiv 0$ and $w$ is constant on $[0, \infty)$. Hence $w \equiv 0$.

The second set of boundary conditions is dealt with in the same manner.

\section{References}

1. S. Agmon, A. Douglis and L. Nirenberg, Estimates near the boundary for solutions of elliptic partial differential equations satisfying general boundary conditions II. Commun. Pure Appl. Math. 17 (1964) 35-92.

2. A.R. Champneys, P.J. McKenna and P.A. Zegeling, Solitary waves in nonlinear beam equations; stability, fission and fusion. Nonlinear Dyn. 21 (2000) 31-53.

3. A.R. Champneys, G.H.M. van der Heijden and J.M.T. Thompson, Spatially complex localization after one-twist-per-wave equilibria in twisted circular rods with initial curcature. Philos. Trans. R. Soc. Lond. A 355 (1997) 2151-2174. See also [4], pp. 157-180.

4. A.R. Champneys, G.W. Hunt and J.M.T. Thompson (eds.), Localization and Solitary Waves in Solid Mechanics. World Sci. Publishing, River Edge, NJ (1999).

5. P.J. Davies, Buckling and barrelling instabilities in finite elasticity. J. Elast. 21 (1989) $147-$ 192.

6. Y.V. Egorov and M.A. Shubin (eds.), Partial Differential Equations I, Encyclopaedia of Mathematical Sciences. Springer, Berlin Heidelberg New York, 30.

7. G. Fichera, Existence theorems in elasticity, Handbuch der Physik, Bd. VIa/2, 347-389. Springer, Berlin Heidelberg New York (1972).

8. Y.B. $\mathrm{Fu}$ and A. Mielke, A new identity for the surface impedance matrix and its application to the determination of surface-wave speeds. Proc. R. Soc. Lond., A 458 (2002) 2523-2543.

9. Y.B. Fu and A. Mielke, Uniqueness of the surface-wave speed: A proof that is independent of the Stroh formalism. Math. Mech. Solids 9 (2004) 5-15.

10. T.H. Healey and E.L. Montes-Pizarro, Global bifurcation in nonlinear elasticity with an application to barrelling states of cylindrical columns. J. Elast. 71 (2003) 33-58.

11. G.W. Hunt, G.J. Lord and A.R. Champneys, Homoclinic and heteroclinic orbits underlying the post-buckling of axially compressed cylindrical shells. Comput. Methods Appl. Mech. Eng. 170 (1999) 239-251. See also [4], pp. 285-297.

12. G.W. Hunt, M.A. Peletier, A.R. Champneys, P.D. Woods, M. Ahmer Wadee, C.J. Budd and G.J. Lord, Cellular buckling in long structures. Nonlinear Dyn. 21 (2000) 3-29.

13. J.K. Knowles and E. Sternberg, On the failure of ellipticity of the equations for finite elastostatic plane strain. Arch. Ration. Mech. Anal. 63 (1977) 321-336.

14. A. Mielke, Hamiltonian and Lagrangian fiows on center manifolds, Lecture Notes in Mathematics. Springer, Berlin Heidelberg New York, 1489, 1991. 
15. A. Mielke, Reduction of quasilinear elliptic equations in cylindrical domains with applications. Math. Methods Appl. Sci. 10 (1988) 51-66.

16. A. Mielke and P. Sprenger, Quasiconvexity at the boundary and a simple formulation of Agmon's condition. J. Elas. 51 (1998) 23-41.

17. P.V. Negrón-Marrero and E.L. Montes-Pizzaro, Axisymmetric deformations of buckling and barreling type for cylinders under lateral compression - the linear problem. J. Elas. 65 (2001) 61-86.

18. H.C. Simpson and S.J. Spector, On barrelling instabilities in finite elasticity. J. Elas. 14 (1984) 103-125.

19. H.C. Simpson and S.J. Spector, On the positivity of the second variation in finite elasticity. Arch. Ration. Mech. Anal. 98 (1987) 1-30. 